\title{
Ganho de peso de porcas gestantes associado ao comportamento em baias e à uniformidade da leitegada
}

\author{
Andrea Panzardi(1), Ana Paula Gonçalves Mellagi(1), Thomas Bierhals ${ }^{(1)}$, Neimar Bonfati Gheller(1), \\ Mari Lourdes Bernardi(2), Fernando Pandolfo Bortolozzo ${ }^{(1)}$ e Ivo Wentz ${ }^{(1)}$
}

(1)Universidade Federal do Rio Grande do Sul (UFRGS), Faculdade de Veterinária, Setor de Suínos, Avenida Bento Gonçalves, no 9.090, CEP 90650-00 Porto Alegre, RS. E-mail: andreapanzardi@gmail.com, paulamellagi@yahoo.com.br, thombier@hotmail.com, neimarvet@yahoo.com.br, fpbortol@ufrgs.br, ivowentz@ufrgs.br (2)UFRGS, Departamento de Zootecnia, Avenida Bento Gonçalves, no 7.712, CEP 91540-000 Porto Alegre, RS. E-mail: mari.bernardi@ufrgs.br

\begin{abstract}
Resumo - O objetivo deste trabalho foi avaliar a associação do comportamento de fêmeas suínas mantidas em baias coletivas, durante a alimentação, com o ganho de peso no último mês de gestação, e determinar se a uniformidade de peso das leitegadas é influenciada pelo ganho de peso das fềmeas. As fềmeas $(n=699)$ foram separadas em três grupos de ordem de parto (OP): 2, 3-5 e 6-9. Cada grupo de OP foi dividido em três subgrupos com percentual de ganho de peso: baixo, médio e alto. Fêmeas de OP 6-9 tiveram mais leitões com peso menor que $1.200 \mathrm{~g}$ e maior coeficiente de variação desse peso, em comparação às de OP 2. O menor peso de leitões e o maior número de leitões com peso $<1.200 \mathrm{~g}$ foram observados no subgrupo de peso baixo. Houve correlação do percentual de ganho de peso na gestação com o número de vezes que a fêmea esteve em pé no cocho $(\mathrm{r}=0,669)$ e com o número de vezes que a fêmea agrediu outras fêmeas $(\mathrm{r}=0,451)$. A variação do ganho de peso das fêmeas, durante o último mês de gestação, em baias coletivas é influenciada pela competição durante a alimentação, e o menor ganho de peso no último mês de gestação reduz o peso dos leitões ao nascimento.
\end{abstract}

Termos para indexação: alojamento coletivo, fêmeas suínas gestantes, leitões, peso ao nascimento.

\section{Weight gain of pregnant sows associated with their behavior in pens and to the uniformity of piglets}

\begin{abstract}
The objective of this work was to evaluate the association between the behavior of group-housed sows during feeding time, with weight gain during the last month of gestation and to determine if piglet birth weight uniformity is influenced by sow weight gain. Sows $(\mathrm{n}=699)$ were separated into three parity groups $(\mathrm{P}): 2,3-5$, and 6-9. Each parity group was divided in three subgroups according to the percentage of weight gain: low, medium, and high. Sows from P 6-9 showed higher number of piglet birth weight below 1,200 $\mathrm{g}$ and higher birth weight coefficient of variation, in comparison to $\mathrm{P} 2$ sows. Lower birth weight piglets and higher number of piglets weighing $<1,200 \mathrm{~g}$ were observed in the low weight subgroup. There was a correlation of weight gain percentage during gestation with the number of visits to the feeder $(r=0.669)$ and with the number of aggressive encounters $(r=0.451)$. Variation in weight gain during the last month of gestation in group-housed sows is influenced by competition at feeding time, and the lower weight gain during the last month of gestation reduces the birth weight of piglets.
\end{abstract}

Index terms: group-housing, pregnant sows, piglets, birth weight.

\section{Introdução}

A domesticação dos suínos fez com que suas características gregárias fossem parcialmente perdidas e, com o advento do sistema de alojamento em gaiolas individuais, as fêmeas suínas eliminaram, em grande parte, suas características naturais de comportamento. A agressividade na espécie suína está diretamente relacionada à necessidade de formação de um grupo social, em que é determinada a ordem hierárquica de cada indivíduo (Séguin et al., 2006). Estudos relacionados aos tipos de alojamentos e, principalmente, ao bem-estar animal têm sido, mundialmente, discutidos nos meios técnico, científico e acadêmico (Séguin et al., 2006), com grande interesse de países da Europa e da América do Norte (Remience et al., 2008).

Atualmente o tipo de alojamento mais utilizado, para fêmeas gestantes e desmamadas, é o de gaiolas individuais, amplamente usado em diversos países (Hulbert \& Mcglone, 2006; Salak-Johnson et al., 2007). Esse sistema permite a individualização do arraçoamento e facilita o diagnóstico do estro, e posterior inseminação artificial (Jansen et al., 2007).

Pesq. agropec. bras., Brasília, v.46, n.11, p.1562-1569, nov. 2011 
No entanto, gera diferentes níveis de estresse, comportamentos agressivos (Løvendahl et al., 2005) e estereotipias (María Levrino \& Villarroel Robinson, 2003). Além disso, as gaiolas individuais não permitem a expressão normal de comportamento das fêmeas, como o de girar em torno de seu próprio eixo e as interações sociais (Hulbert \& Mcglone, 2006).

Em contrapartida, as baias coletivas permitem interações sociais, que podem causar problemas de consumo de alimento e, em consequência, problemas reprodutivos (Spoolder et al., 2009). As baias coletivas devem ter espaço suficiente para permitir o acesso à quantidade necessária de alimento, além de permitir um comportamento de fuga na área do cocho, onde ocorre grande número de disputas (Remience et al., 2008).

Como $60 \%$ do crescimento fetal ocorre no terço final da gestação (Noblet et al., 1990), é necessário o aporte de nutrientes adequado, nessa fase, para garantir bom peso dos leitões ao nascimento. A maioria dos trabalhos de pesquisa são relacionados ao tipo de alojamento no terço inicial (Kranendonk et al., 2007; Remience et al., 2008) ou médio da gestação (Séguin et al., 2006; Jansen et al., 2007), e poucos estão relacionados a fêmeas mantidas em alojamento coletivo no terço final da gestação, com avaliação de ganho de peso e desempenho produtivo.

O objetivo deste trabalho foi avaliar, a associação do comportamento de fêmeas suínas, mantidas em baias coletivas durante a alimentação, com ganho de peso no último mês de gestação, e determinar se a uniformidade de peso das leitegadas é influenciada pelo ganho de peso das fêmeas.

\section{Material e Métodos}

$\mathrm{O}$ experimento foi realizado em uma granja comercial com capacidade para 5.500 matrizes, de janeiro a maio de 2008, em Diamantino, MT (14² $24^{\prime} 11^{\prime \prime S}$ e $56^{\circ} 25^{\prime} 37^{\prime \prime} \mathrm{W}$, a $344 \mathrm{~m}$ de altitude). Foram utilizadas 699 fêmeas da genética Agroceres PIC, linhagem Camborough 23, e ordem de parto (OP) de 2 a 9 , acompanhadas entre 80 e 85 dias de gestação até o pós-parto. No dia anterior à transferência para as baias de gestação coletiva, foram registrados os dados referentes à $\mathrm{OP}$, espessura de toucinho (ET) no ponto P2, em ambos os lados, e detecção de prenhez, utilizando aparelho ultrassom em tempo real (Aloka Co. Ltd., Mure, Mitaka-shi, Tóquio, Japão), com transdutor curvilinear com frequência de $5 \mathrm{MHz}$ US, pelo método transabdominal. As fêmeas foram pesadas, individualmente, por ocasião da transferência para as baias de gestação coletiva.

Das 752 fêmeas avaliadas, 616 estavam agrupadas em baias com quatro fêmeas (G4) e 136 em baias com oito (G8). Entretanto, somente 699 fêmeas foram incluídas nas análises (574 e 125 para G4 e G8, respectivamente), pois não foi possível obter o peso de todos os leitões, por ocasião do parto de 53 fêmeas (42 G4 e 11 G8). As baias G4 e os cochos mediam 4,67x2,30 m e 0,28x1,40 m de comprimento e largura, respectivamente. Cada cocho continha quatro divisórias medindo $0,35 \times 0,35 \times 0,24 \mathrm{~m}$ de comprimento, largura e altura, respectivamente, e lâmina de água de $0,80 \times 2,30 \times 0,10 \mathrm{~m}$ de comprimento, largura e profundidade, respectivamente. As baias G8 tinham o mesmo comprimento e o dobro da largura das baias G4. O espaço disponível era de $2,7 \mathrm{~m}^{2}$ por fêmea, tanto nas baias G4 quanto nas baias G8.

Depois de alojadas, as fêmeas foram identificadas com um número na vértebra cervical. A ração foi fornecida duas vezes ao dia, às $7 \mathrm{~h} 30$ e às $15 \mathrm{~h}$, com $12 \mathrm{~kg}$ de ração para as fêmeas da baia G4 e $24 \mathrm{~kg}$ para as da G8. A quantidade diária de ração pré-lactação estabelecida para cada fêmea foi de $3 \mathrm{~kg}$, com $3.150 \mathrm{kcal} \mathrm{kg}^{-1}$ de EM, 17\% PB e 0,9\% lisina. Para minimizar as disputas, em cada refeição, parte da ração foi colocada no cocho $(\sim 70 \%)$ e parte no chão, na parte lateral da baia $(\sim 30 \%)$.

A avaliação do comportamento das fêmeas foi iniciada no terceiro dia de alojamento. As fêmeas de cada baia foram avaliadas quatro vezes, com intervalo de uma semana entre cada avaliação. Foram realizadas as seguintes avaliações de comportamento das fêmeas em três momentos do arraçoamento (0,10 e $20 \mathrm{~min})$ : localização da fêmea, se ela estava em pé no cocho ou na lateral da baia onde o alimento havia sido distribuído; se ela agrediu outras fêmeas, e, o grau das lesões, de acordo com a escala: 0, ausência de lesão; 1 , lesões em pequeno número, de baixa gravidade; 2 , lesões em número médio, de gravidade média; 3 , lesões em grande número, de alta gravidade.

Entre 105 e 111 dias de gestação, as fêmeas foram transferidas para a maternidade. Antes da transferência elas foram pesadas e a ET foi medida, individualmente. Após o parto, foram anotados os números de leitões nascidos totais (NT), nascidos vivos (NV), natimortos (NAT) e mumificados (MUM), e foram pesados individualmente os NV e NAT. 
As análises estatísticas foram realizadas pelo SAS (SAS Institute, 2005). Foi efetuada a análise das características corporais das fêmeas no dia da entrada no alojamento coletivo, ganho de peso durante $o$ alojamento coletivo e desempenho no parto de três grupos, separados de acordo com as OP 2, 3-5 e 6-9. Os dados das fêmeas, mantidas nas baias G4 e G8, foram analisados em conjunto, pois foi verificado que o ganho de peso, no último mês de gestação, não foi influenciado pelo tipo de baia, em nenhum dos grupos de OP. Dessa forma, para análise, esses grupos foram subdivididos nos subgrupos: baixo, médio e alto, de acordo com o percentual de ganho de peso, no último mês de gestação. Os limites dos percentuais de cada subgrupo foram diferentes entre as classes de OP, pois dentro de cada classe de OP, foi distribuído em subgrupos um percentual de fêmeas próximo de $33 \%$.

As variáveis: peso no início do alojamento coletivo; coeficiente de variação (CV) do peso de entrada nas baias coletivas; ganho de peso ajustado para 28 dias de gestação; percentual de ganho de peso; ET; número de NT e de NV; peso médio dos leitões; CV do peso dos leitões e número de leitões, com peso menor que $1.200 \mathrm{~g}$, foram submetidas à análise de variância pelo procedimento GLM do SAS, e comparadas pelo teste de Tukey-Kramer, a 5\% de probabilidade. O percentual de leitões natimortos, de fetos mumificados, número de vezes em que a fêmea ficou de pé no cocho e o número de vezes que ela agrediu outra fêmea foram analisados pelo procedimento NPAR1WAY do SAS, e a comparação entre os grupos ou subgrupos foi realizada pelo teste de Kruskal-Wallis, a 5\% de probabilidade. Associações entre o percentual de ganho de peso das fêmeas, em 28 dias de alojamento coletivo, e as variáveis de comportamento das fêmeas foram analisadas pela correlação de Spearman, pelo procedimento CORR, do SAS.

\section{Resultados e Discussão}

Na época do alojamento coletivo, as fêmeas OP 2 tinham menos peso que as fêmeas de OP 3-5 e 6-9 (Tabela 1). O percentual de ganho de peso, durante os 28 dias de gestação, foi maior $(\mathrm{p}<0,05)$ nos grupos de OP 2 e 3-5 do que no de OP 6-9. O fato de as fêmeas mais jovens (OP 2) apresentarem maior ganho de peso no último mês de gestação, em comparação às fêmeas mais velhas (OP 6-9), era de se esperar, uma vez que elas ainda não haviam atingido o tamanho adulto.
Fêmeas de primeiro e segundo partos devem apresentar maior ganho de peso, em relação às fêmeas de terceiro ou mais partos, para atingir a condição corporal desejada ao parto (Young et al., 2005). Esse fato é explicado pela diferença na composição de ganho em proteína, que constitui a maior proporção do ganho de peso materno em fêmeas mais jovens.

O final da gestação é período de maior crescimento e desenvolvimento dos fetos, época em que as fêmeas apresentam maior ganho de peso (Noblet et al., 1997), o que foi confirmado no presente trabalho, em que fêmeas de OP 2 e 3-5 ganharam mais da metade do ganho total de $45 \mathrm{~kg}$, em 28 dias de gestação coletiva (Tabela 1), o que era previsto para acontecer durante a primeira gestação (National Research Council, 1998). Maior quantidade de nutrientes é necessária no final da gestação, pois o conteúdo de proteína e de gordura nos fetos aumenta de forma quadrática à medida que a gestação avança. Antes de 69 dias de gestação, a deposição de proteína e de gordura nos fetos é de, respectivamente, 0,25 e $0,06 \mathrm{~g}$ por dia e aumenta para 4,63 e 1,09 g por dia, após 69 dias de gestação (McPherson et al., 2004).

Foi observada correlação positiva $(p<0,0001)$ entre o percentual de ganho de peso na gestação e o número de vezes em que as fêmeas alimentaram-se no cocho $(\mathrm{r}=0,669)$, e o número de vezes que elas agrediram outras fêmeas $(r=0,451)$ e correlação negativa $(p<0,0001)$ entre o percentual de ganho de peso e o grau de lesões $(r=-0,338)$. Em baias com formato retangular, tem sido relatado menor índice de agressões em comparação às baias de formato quadrado (Barnett et al., 1993) ou redondo (Wiegand et al., 1994). Apesar de as baias serem retangulares e o espaço disponível por fềmea $\left(2,7 \mathrm{~m}^{2}\right)$ ter sido acima do espaço mínimo determinado pela União Europeia $\left(2,25 \mathrm{~m}^{2}\right)$, é provável que a infraestrutura das baias do presente trabalho, com relação à disposição e espaço nos cochos, tenha propiciado maior disputa entre as fêmeas no momento do arraçoamento e, consequentemente, maior número de agressões por parte daquelas de maior ganho de peso (Tabelas 2, 3 e 4). A associação entre ganho de peso, no final da gestação, e comportamento das fêmeas, durante a alimentação, também é reforçada pela maior presença, no cocho, de fêmeas do subgrupo alto ganho de peso, em comparação àquelas com baixo e médio ganho de peso. Kranendonk et al. (2007) também observaram que o nível hierárquico de fêmeas gestantes, no seu 
grupo social, está associado à competição por alimento, uma vez que aquelas com menor ganho de peso foram as que tiveram menos acesso ao comedouro.
A maior uniformidade de peso de leitões ao nascimento (menor $\mathrm{CV}$ ), em fêmeas de OP 2, em comparação às fêmeas de $\mathrm{OP} 3-5$, deve estar relacionada

Tabela 1. Variáveis corporais de fêmeas suínas alojadas em baias coletivas, entre 83 e 111 dias de gestação, e de leitegada, em relação à ordem de parto.

\begin{tabular}{|c|c|c|c|}
\hline \multirow[t]{2}{*}{ Variável } & \multicolumn{3}{|c|}{ Ordem de parto } \\
\hline & $2(n=137)$ & $3-5(n=391)$ & $6-9(n=171)$ \\
\hline & \multicolumn{3}{|c|}{ Variáveis corporais de fêmeas ${ }^{(1)}$} \\
\hline Peso aos 83 dias de gestação $(\mathrm{kg})$ & $209,6 \pm 1,42 \mathrm{a}$ & $245,1 \pm 1,15 b$ & $276,1 \pm 1,75 \mathrm{c}$ \\
\hline Peso aos 111 dias de gestação (kg) & $233,3 \pm 1,86 \mathrm{a}$ & $271,1 \pm 1,47 \mathrm{~b}$ & $291,3 \pm 2,43 c$ \\
\hline Ganho de peso em 28 dias de gestação $(\mathrm{kg})$ & $23,5 \pm 1,35 \mathrm{a}$ & $25,9 \pm 0,99 \mathrm{a}$ & $15,0 \pm 1,80 \mathrm{~b}$ \\
\hline Percentual de ganho de peso & $11,3 \pm 0,66 \mathrm{a}$ & $10,7 \pm 0,41 \mathrm{a}$ & $5,5 \pm 0,66 \mathrm{~b}$ \\
\hline $\mathrm{ET}^{(2)}$ aos 83 dias de gestação & $14,6 \pm 0,25 \mathrm{a}$ & $15,0 \pm 0,17 \mathrm{a}$ & $15,4 \pm 0,32 \mathrm{a}$ \\
\hline \multirow[t]{2}{*}{ ET aos 111 dias de gestação } & $15,0 \pm 0,30 \mathrm{a}$ & $15,6 \pm 0,22 \mathrm{a}$ & $14,9 \pm 0,36 \mathrm{a}$ \\
\hline & \multicolumn{3}{|c|}{ Variáveis da leitegada } \\
\hline Número total de leitões nascidos ${ }^{(1)}$ & $11,8 \pm 0,27 \mathrm{a}$ & $12,8 \pm 0,17 \mathrm{~b}$ & $12,2 \pm 0,24 \mathrm{ab}$ \\
\hline Número de leitões nascidos vivos ${ }^{(1)}$ & $11,0 \pm 0,25 \mathrm{a}$ & $12,0 \pm 0,15 b$ & $11,2 \pm 0,21 \mathrm{a}$ \\
\hline Natimortos $(\%)^{(3)}$ & $3,57 \pm 0,51 \mathrm{a}$ & $4,12 \pm 0,33 \mathrm{a}$ & $5,03 \pm 0,57 \mathrm{a}$ \\
\hline Mumificados $(\%)^{(3)}$ & $3,03 \pm 0,50 \mathrm{a}$ & $2,07 \pm 0,24 \mathrm{a}$ & $2,42 \pm 0,34 \mathrm{a}$ \\
\hline Peso médio dos leitões $(\mathrm{g})^{(1)}$ & $1.532 \pm 20,65 \mathrm{a}$ & $1.489 \pm 12,22 \mathrm{ab}$ & $1.462 \pm 18,48 \mathrm{~b}$ \\
\hline Número de leitões com peso $<1.200 \mathrm{~g}^{(1)}$ & $2,1 \pm 0,25 \mathrm{a}$ & $3,1 \pm 0,14 \mathrm{~b}$ & $3,4 \pm 0,22 \mathrm{~b}$ \\
\hline$\overline{\mathrm{CV}}$ do peso ao nascimento $(\%)^{(1)}$ & $17,8 \pm 0,59 a$ & $21,0 \pm 0,35 b$ & $22,5 \pm 0,53 \mathrm{c}$ \\
\hline
\end{tabular}

${ }^{(1)}$ Médias seguidas de letras diferentes, na linha, diferem entre si pelo teste Tukey-Kramer a $5 \%$ de probabilidade. ${ }^{(2)}$ ET, espessura de toucinho. ${ }^{(3)}$ Médias seguidas de letras diferentes, na linha, diferem entre si pelo teste de Kruskal-Wallis a $5 \%$ de probabilidade.

Tabela 2. Variáveis corporais e comportamentais de fêmeas suínas de ordem de parto 2 no início do alojamento, em baias coletivas e da leitegada, em relação a três classes de ganho de peso: baixo (até 8,5\%), médio $(8,6-14,5 \%)$, alto (>14,5\%), entre 83 e 111 dias de gestação.

\begin{tabular}{|c|c|c|c|}
\hline Variável & Baixo $(n=47)$ & Médio $(n=43)$ & Alto $(n=47)$ \\
\hline & \multicolumn{3}{|c|}{ Variáveis corporais das fêmeas $^{(1)}$} \\
\hline Peso aos 83 dias de gestação $(\mathrm{kg})$ & $215,8 \pm 2,17 \mathrm{a}$ & $209,2 \pm 2,09 \mathrm{ab}$ & $203,9 \pm 2,72 b$ \\
\hline CV do peso aos 83 dias de gestação (\%) & $6,9 \pm 0,34 \mathrm{a}$ & $6,6 \pm 0,39 \mathrm{a}$ & $6,9 \pm 0,36 \mathrm{a}$ \\
\hline Peso aos 111 dias de gestação $(\mathrm{kg})$ & $222,8 \pm 2,96 a$ & $233,2 \pm 2,41 \mathrm{~b}$ & $243,9 \pm 3,36 c$ \\
\hline Ganho de peso em 28 dias de gestação $(\mathrm{kg})$ & $7,0 \pm 1,50 \mathrm{a}$ & $23,8 \pm 0,58 \mathrm{~b}$ & $39,7 \pm 1,21 \mathrm{c}$ \\
\hline Percentual de ganho de peso & $3,2 \pm 0,69 \mathrm{a}$ & $11,4 \pm 0,25 b$ & $19,5 \pm 0,60 \mathrm{c}$ \\
\hline $\mathrm{ET}^{(2)}$ aos 83 dias de gestação & $15,1 \pm 0,40 \mathrm{a}$ & $15,1 \pm 0,46 \mathrm{a}$ & $13,5 \pm 0,39 \mathrm{~b}$ \\
\hline \multirow[t]{2}{*}{ ET aos 111 dias de gestação } & $13,6 \pm 0,44 \mathrm{a}$ & $15,3 \pm 0,56 \mathrm{~b}$ & $16,0 \pm 0,48 \mathrm{~b}$ \\
\hline & \multicolumn{3}{|c|}{ Variáveis comportamentais das fêmeas ${ }^{(3)}$} \\
\hline Número de vezes em pé no cocho(4) & $3,1 \pm 0,29 \mathrm{a}$ & $5,2 \pm 0,41 \mathrm{~b}$ & $7,8 \pm 0,48 \mathrm{c}$ \\
\hline Número de vezes em que a fêmea agrediu & $0,57 \pm 0,15 \mathrm{a}$ & $0,37 \pm 0,09 \mathrm{a}$ & $1,87 \pm 0,31 \mathrm{~b}$ \\
\hline \multirow[t]{2}{*}{ Grau de lesões ${ }^{(5)}$} & $1,08 \pm 0,06 \mathrm{a}$ & $0,83 \pm 0,06 \mathrm{~b}$ & $0,64 \pm 0,06 \mathrm{c}$ \\
\hline & \multicolumn{3}{|c|}{ Variáveis da leitegada } \\
\hline Número total de leitões nascidos ${ }^{(1)}$ & $11,9 \pm 0,45 \mathrm{a}$ & $11,7 \pm 0,43 \mathrm{a}$ & $11,8 \pm 0,53 \mathrm{a}$ \\
\hline Número de leitões nascidos vivos ${ }^{(1)}$ & $10,8 \pm 0,41 \mathrm{a}$ & $11,0 \pm 0,39 \mathrm{a}$ & $11,1 \pm 0,49 \mathrm{a}$ \\
\hline Natimortos $(\%)^{(3)}$ & $5,14 \pm 1,03 \mathrm{a}$ & $1,39 \pm 0,51 b$ & $4,00 \pm 0,88 \mathrm{a}$ \\
\hline Mumificados $(\%)^{(3)}$ & $3,69 \pm 0,81 \mathrm{a}$ & $3,82 \pm 1,16 \mathrm{a}$ & $1,65 \pm 0,58 \mathrm{a}$ \\
\hline Peso médio dos leitões $(\mathrm{g})^{(1)}$ & $1.479 \pm 33,80 \mathrm{a}$ & $1.598 \pm 33,71 \mathrm{~b}$ & $1.527 \pm 36,53 \mathrm{ab}$ \\
\hline Número de leitões com peso $<1.200 \mathrm{~g}^{(1)}$ & $2,7 \pm 0,42 \mathrm{a}$ & $1,5 \pm 0,25 \mathrm{~b}$ & $2,2 \pm 0,31 \mathrm{ab}$ \\
\hline CV do peso ao nascimento $(\%)^{(1)}$ & $18,8 \pm 1,11 \mathrm{a}$ & $16,0 \pm 0,77 \mathrm{a}$ & $18,4 \pm 0,91 \mathrm{a}$ \\
\hline
\end{tabular}

${ }^{(1)}$ Médias seguidas de letras diferentes, na linha, diferem entre si pelo teste Tukey-Kramer, a $5 \%$ de probabilidade. (2)ET, espessura de toucinho. ${ }^{(3)}$ Médias seguidas de letras diferentes, na linha, diferem entre si pelo teste de Kruskal-Wallis, a 5\% de probabilidade. ${ }^{(4)}$ Valor máximo corresponde a 12 , derivado de quatro avaliações, efetuadas com intervalo de uma semana, em três momentos da refeição (no momento da oferta do alimento, aos 10 e 20 min após a oferta). ${ }^{(5)}$ Valor máximo corresponde a 3, com base na escala: 0 , ausência de lesão; 1 , lesões em pequeno número, de baixa gravidade; 2 , lesões em número médio, de gravidade média; 3 , lesões em grande número, de alta gravidade. 
ao menor número de NT observado nas fêmeas de OP 2 (Tabela 1), o que confirma a associação existente entre tamanho da leitegada e peso dos leitões (Quiniou et al., 2002). No entanto, fêmeas de OP 6-9 tiveram tamanho de leitegadas semelhante às das fêmeas de OP 2, mas com maior $\mathrm{CV}$ de peso ao nascimento e maior número de leitões com peso menor que $1.200 \mathrm{~g}$ e menor peso médio dos leitões (Tabela 1). Esse fato provavelmente está relacionado ao menor ganho de peso ou, mesmo, à perda de peso de cerca de um terço das fêmeas de OP 6-9, no último mês de gestação (Tabela 4). Neste caso, mesmo com a mobilização de reservas nutricionais das fêmeas, houve redução no peso dos leitões, o que confirma que a suplementação inadequada pode resultar em 15 a $20 \%$ de leitões com baixo peso, cuja sobrevivência e desenvolvimento pós-natal ficam comprometidos (Pettigrew Junior, 1981). Apesar de as fêmeas de maior OP possuírem maior massa corpórea e mais reservas nutricionais, há evidências de que a restrição alimentar durante o terço final da gestação resulta em menor peso de leitões ao nascimento, uma vez que elas são pouco adaptáveis às mudanças nos níveis nutricionais, quando comparadas às fêmeas jovens (Pluske et al., 1995).

Não foi observada diferença do $\mathrm{CV}$ de peso, ao nascimento $(p>0,05)$, entre leitões dos subgrupos de ganho de peso, em todas as classes de OP (Tabelas 2, 3 e 4). O subgrupo médio ganho de peso na gestação, obteve maior peso de leitões e menor número de leitões com peso menor que $1.200 \mathrm{~g}(\mathrm{p}<0,05)$ do que o subgrupo baixo ganho de peso na gestação, tanto em fêmeas de OP 2 (Tabela 2) quanto em fêmeas de OP 3-5 (Tabela 3). Nas fêmeas de OP 6-9, o subgrupo alto ganho de peso na gestação apresentou maior peso dos leitões $(p<0,05)$ do que os subgrupos médio e baixo (Tabela 4). Esses resultados sugerem que nem sempre o maior ganho de peso das fêmeas resulta em maior peso dos leitões. Se for considerado o ganho de peso do subgrupo baixo, que obteve menor peso dos leitões, em todas as classes de OP, em comparação ao subgrupo médio ou ao subgrupo alto, pode-se concluir que as fêmeas necessitam ganhar mais de 500 g por dia, no último mês da gestação. Caso contrário, a ingestão de alimento será insuficiente para suprir as necessidade nutricionais do feto. Em estudos

Tabela 3. Variáveis corporais e comportamentais de fêmeas suínas de ordem de parto 3 a 5 , no início do alojamento, em baias coletivas, e da leitegada, em relação a três classes de ganho de peso: baixo (até 6,5\%), médio (6,6-14\%), alto (>14\%), entre 83 e 111 dias de gestação.

\begin{tabular}{|c|c|c|c|}
\hline Variável & Baixo $(n=124)$ & Médio $(n=130)$ & Alto $(n=137)$ \\
\hline \multicolumn{4}{|c|}{ Variáveis corporais das fêmeas $^{(1)}$} \\
\hline Peso aos 83 dias de gestação $(\mathrm{kg})$ & $249,0 \pm 2,21 \mathrm{a}$ & $245,2 \pm 1,89 \mathrm{ab}$ & $241,3 \pm 1,86 b$ \\
\hline CV do peso aos 83 dias de gestação (\%) & $6,0 \pm 0,23 a$ & $5,8 \pm 0,20 \mathrm{a}$ & $6,2 \pm 0,20 \mathrm{a}$ \\
\hline Peso aos 111 dias de gestação $(\mathrm{kg})$ & $252,6 \pm 2,26 \mathrm{a}$ & $270,9 \pm 2,18 \mathrm{~b}$ & $288,0 \pm 2,20 \mathrm{c}$ \\
\hline Ganho de peso em 28 dias de gestação $(\mathrm{kg})$ & $3,6 \pm 0,88 \mathrm{a}$ & $25,4 \pm 0,53 b$ & $46,6 \pm 0,84 \mathrm{c}$ \\
\hline Percentual de ganho de peso & $1,5 \pm 0,35 \mathrm{a}$ & $10,4 \pm 0,19 b$ & $19,4 \pm 0,36 \mathrm{c}$ \\
\hline $\mathrm{ET}^{(2)}$ aos 83 dias de gestação & $14,8 \pm 0,32 \mathrm{a}$ & $15,0 \pm 0,29 \mathrm{a}$ & $15,2 \pm 0,31 \mathrm{a}$ \\
\hline \multirow[t]{2}{*}{ ET aos 111 dias de gestação } & $13,5 \pm 0,34 \mathrm{a}$ & $15,6 \pm 0,36 \mathrm{~b}$ & $17,5 \pm 0,37 \mathrm{c}$ \\
\hline & \multicolumn{3}{|c|}{ Variáveis comportamentais das fêmeas ${ }^{(3)}$} \\
\hline Número de vezes em pé no $\operatorname{cocho}^{(4)}$ & $2,1 \pm 0,20 \mathrm{a}$ & $4,2 \pm 0,27 \mathrm{~b}$ & $8,2 \pm 0,30 \mathrm{c}$ \\
\hline Número de vezes em que a fêmea agrediu & $0,51 \pm 0,08 \mathrm{a}$ & $0,99 \pm 0,12 b$ & $2,08 \pm 0,19 \mathrm{c}$ \\
\hline \multirow[t]{2}{*}{ Grau de lesões $^{(5)}$} & $0,92 \pm 0,04 \mathrm{a}$ & $0,83 \pm 0,04 \mathrm{a}$ & $0,53 \pm 0,03 b$ \\
\hline & \multicolumn{3}{|c|}{ Variáveis da leitegada } \\
\hline Número total de leitões nascidos ${ }^{(1)}$ & $12,6 \pm 0,30 \mathrm{a}$ & $12,8 \pm 0,29 \mathrm{a}$ & $13,0 \pm 0,27 \mathrm{a}$ \\
\hline Número de leitões nascidos vivos ${ }^{(1)}$ & $11,8 \pm 0,29 \mathrm{a}$ & $11,9 \pm 0,27 \mathrm{a}$ & $12,1 \pm 0,24 \mathrm{a}$ \\
\hline Natimortos $(\%)^{(3)}$ & $4,09 \pm 0,63 \mathrm{a}$ & $3,91 \pm 0,56 \mathrm{a}$ & $4,34 \pm 0,52 \mathrm{a}$ \\
\hline Mumificados $(\%)^{(3)}$ & $2,06 \pm 0,48 \mathrm{a}$ & $2,36 \pm 0,39 \mathrm{a}$ & $1,82 \pm 0,38 \mathrm{a}$ \\
\hline Peso médio dos leitões $(\mathrm{g})^{(1)}$ & $1.456 \pm 21,19 \mathrm{a}$ & $1.518 \pm 21,75 b$ & $1.493 \pm 19,82 \mathrm{ab}$ \\
\hline Número de leitões com peso $<1.200 \mathrm{~g}^{(1)}$ & $3,5 \pm 0,30 \mathrm{a}$ & $2,7 \pm 0,24 b$ & $3,1 \pm 0,22 \mathrm{ab}$ \\
\hline CV do peso ao nascimento $(\%)^{(1)}$ & $21,4 \pm 0,61 \mathrm{a}$ & $20,1 \pm 0,62 \mathrm{a}$ & $21,6 \pm 0,60 \mathrm{a}$ \\
\hline
\end{tabular}


que classifica a hierarquia de fêmeas gestantes de acordo com sua capacidade de deslocamento no momento do acesso ao comedouro, foi observado menor ganho de peso na gestação e tendência de menor peso dos leitões, ao nascimento, naquelas de baixo escore hierárquico, em comparação àquelas de alto escore (Kranendonk et al., 2007).

O ganho de peso próximo ou maior que $1.400 \mathrm{~g}$ por dia, observado no subgrupo alto, em fêmeas de OP 2 e 3-5, não aumentou o peso dos leitões. Parece ter havido desvio de nutrientes para ganho de peso e engorda da fêmea no subgrupo alto, pois ocorreu ganho de $2,5 \mathrm{~mm}$ de ET nas fêmeas de OP 2 (Tabela 2) e de 2,3 mm nas fêmeas de OP 3-5 (Tabela 3), durante o último mês de gestação. Nas fêmeas de OP 2 e 3-5, o subgrupo médio (ganho de peso diário de 800 a 900 g) apresentou melhores resultados em peso dos leitões ao nascimento. Nas fêmeas mais velhas (OP 6-9), o ganho de peso de mais de $1.400 \mathrm{~g}$ por dia, observado no subgrupo alto, foi revertido em maior peso dos leitões (Tabela 4). Apesar de as fêmeas de OP 6-9 terem maior peso corporal, a quantidade de ração fornecida foi semelhante à das fêmeas das outras OP. Não foi possível controlar e medir o consumo individual de ração; provavelmente as fêmeas de OP 6-9, com menor acesso ao cocho, não conseguiram ingerir quantidade de ração necessária para mantença do peso corporal e dos fetos, entrando, assim, em processo de catabolismo durante a gestação (Miller et al., 2000).

Não foi observada diferença no total de leitões nascidos e de leitões nascidos vivos $(p>0,05)$ entre os subgrupos de ganho de peso, em todas as classes de OP (Tabelas 2, 3 e 4). O menor percentual de natimortos no subgrupo médio, nas fêmeas de OP 2 (Tabela 2), pode estar relacionado ao fato de elas terem tido menor número de leitões com peso inferior a $1.200 \mathrm{~g}$. Isto pode ter sido decorrente da adequada ingestão de alimento nesse subgrupo, o que fez com que as fêmeas não chegassem ao final da gestação com peso muito abaixo ou acima do esperado, o que poderia aumentar o número de natimortos (Amaral Filha et al., 2010). Entretanto, o maior percentual de mumificação observado no subgrupo baixo ganho de peso, nas fêmeas de OP 6-9, não está claro, pois a perda de peso

Tabela 4. Variáveis corporais e comportamentais de fêmeas suínas de ordem de parto 6 a 9 , no início do alojamento, em baias coletivas e da leitegada em relação às classes de peso: baixo (até 1,5\%), médio (1,6-7,9\%), alto (>7,9\%), entre 83 e 111 dias de gestação.

\begin{tabular}{|c|c|c|c|}
\hline Variável & Baixo $(n=54)$ & Médio $(n=60)$ & Alto $(n=57)$ \\
\hline \multicolumn{4}{|c|}{ Variáveis corporais das fêmeas $^{(1)}$} \\
\hline Peso aos 83 dias de gestação (kg) & $279,6 \pm 2,67 a$ & $276,1 \pm 2,87 \mathrm{a}$ & $272,7 \pm 3,44 a$ \\
\hline CV do peso aos 83 dias de gestação (\%) & $5,8 \pm 0,33 \mathrm{a}$ & $5,4 \pm 0,28 \mathrm{a}$ & $5,8 \pm 0,32 \mathrm{a}$ \\
\hline Peso aos 111 dias de gestação $(\mathrm{kg})$ & $268,8 \pm 3,10 \mathrm{a}$ & $290,0 \pm 3,20 \mathrm{~b}$ & $313,9 \pm 4,06 \mathrm{c}$ \\
\hline Ganho de peso em 28 dias de gestação $(\mathrm{kg})$ & $-10,8 \pm 1,58 \mathrm{a}$ & $13,8 \pm 0,69 \mathrm{~b}$ & $40,9 \pm 1,85 \mathrm{c}$ \\
\hline Percentual de ganho de peso & $-3,9 \pm 0,58 \mathrm{a}$ & $5,0 \pm 0,24 \mathrm{~b}$ & $15,1 \pm 0,70 \mathrm{c}$ \\
\hline $\mathrm{ET}^{(2)}$ aos 83 dias de gestação & $15,0 \pm 0,47 \mathrm{a}$ & $15,1 \pm 0,54 \mathrm{a}$ & $16,2 \pm 0,64 \mathrm{a}$ \\
\hline \multirow[t]{2}{*}{ ET aos 111 dias de gestação } & $13,7 \pm 0,47 \mathrm{a}$ & $14,3 \pm 0,58 \mathrm{a}$ & $16,8 \pm 0,72 b$ \\
\hline & \multicolumn{3}{|c|}{ Variáveis comportamentais das fêmeas ${ }^{(3)}$} \\
\hline Número de vezes em pé no cocho ${ }^{(4)}$ & $0,93 \pm 0,15 \mathrm{a}$ & $2,4 \pm 0,30 \mathrm{~b}$ & $5,5 \pm 0,47 \mathrm{c}$ \\
\hline Número de vezes em que a fêmea agrediu & $0,28 \pm 0,09 \mathrm{a}$ & $0,65 \pm 0,12 b$ & $2,14 \pm 0,26 \mathrm{c}$ \\
\hline \multirow[t]{2}{*}{ Grau de lesões $^{(5)}$} & $0,85 \pm 0,06 \mathrm{a}$ & $0,84 \pm 0,07 \mathrm{a}$ & $0,59 \pm 0,06 \mathrm{~b}$ \\
\hline & \multicolumn{3}{|c|}{ Variáveis da leitegada } \\
\hline Número total de leitões nascidos ${ }^{(1)}$ & $12,3 \pm 0,42 \mathrm{a}$ & $12,1 \pm 0,38 \mathrm{a}$ & $12,2 \pm 0,44 \mathrm{a}$ \\
\hline Número de leitões nascidos vivos ${ }^{(1)}$ & $11,1 \pm 0,35 \mathrm{a}$ & $11,1 \pm 0,36 \mathrm{a}$ & $11,5 \pm 0,40 \mathrm{a}$ \\
\hline Natimortos $(\%)^{(3)}$ & $5,56 \pm 1,13 \mathrm{a}$ & $5,68 \pm 1,06 \mathrm{a}$ & $3,86 \pm 0,75 \mathrm{a}$ \\
\hline Mumificados $(\%)^{(3)}$ & $3,41 \pm 0,72 \mathrm{a}$ & $2,38 \pm 0,57 \mathrm{ab}$ & $1,53 \pm 0,47 b$ \\
\hline Peso médio dos leitões $(\mathrm{g})^{(1)}$ & $1.415 \pm 35,65 \mathrm{a}$ & $1.451 \pm 31,29 \mathrm{a}$ & $1.519 \pm 31,52 b$ \\
\hline Número de leitões com peso $<1.200 \mathrm{~g}^{(1)}$ & $4,0 \pm 0,53 \mathrm{a}$ & $3,4 \pm 0,38 \mathrm{a}$ & $2,8 \pm 0,32 \mathrm{a}$ \\
\hline CV do peso ao nascimento $(\%)^{(1)}$ & $22,3 \pm 0,92 \mathrm{a}$ & $23,1 \pm 0,89 \mathrm{a}$ & $22,2 \pm 0,93 \mathrm{a}$ \\
\hline
\end{tabular}


dessas fêmeas parece ter ocorrido somente no último mês de gestação, uma vez que, aos 83 dias de gestação, apresentavam peso semelhante ao das fêmeas de outros subgrupos (Tabela 4).

Tem sido relatado na literatura que a simulação de estresse pela administração de acetato de hidrocortisona, em fêmeas gestantes (Kranendonk et al., 2006), ou menor acesso ao comedouro (Kranendonk et al., 2007) afetam negativamente o peso de leitões ao nascimento. É possível que as disputas por alimento, que causou acentuada perda de peso $(-10,8 \mathrm{~kg})$ nesse subgrupo de fêmeas, possa ter gerado estresse pré-natal capaz de levar ao subdesenvolvimento dos fetos e inclusive à morte, o que poderia explicar a maior ocorrência de mumificação.

Embora o alojamento coletivo permita que a fêmea expresse seu comportamento de maneira mais natural, sabe-se que há menor controle do consumo individual de alimento, o que pode gerar grande variação na condição corporal. Além de prejudicar o desenvolvimento do feto, o menor consumo de nutrientes também pode comprometer o desenvolvimento da glândula mamária, com consequente diminuição da produção de leite. Fêmeas em condição corporal inadequada podem ter falhas reprodutivas subsequentes, o que irá aumentar as chances de serem removidas do plantel (Lucia et al., 2000). Ao se optar pelo alojamento coletivo, é importante levar em consideração o sistema de arraçoamento a ser utilizado, que deve ser planejado de forma a reduzir a competição por alimento entre os animais, para evitar a agressividade intensa, além de ser de fácil manejo. Segundo Kranendonk et al. (2007), baias coletivas com o sistema de comedouro automático permitem que fêmeas dominantes desloquem as fêmeas submissas, quando elas estão se alimentando, uma vez que não há proteção individual no momento do arraçoamento. Nesse sistema, é importante ter baias com infraestrutura adequada que considere o tipo de sistema de arraçoamento a ser instituído, de forma que seja permitido o fácil acesso ao alimento pelas fêmeas, principalmente no período do terço final de gestação, momento em que há um crescimento exponencial dos leitões.

\section{Conclusões}

1. O alojamento em baias coletivas permite que algumas fêmeas suínas monopolizem o comedouro durante o arraçoamento.
2. O comportamento de fêmeas suínas no momento do arraçoamento está associado a diferenças no ganho de peso, entre fêmeas de ordem de parto semelhante.

3. Menor peso ao nascimento e maior número de leitões com peso inferior a $1.200 \mathrm{~g}$ ocorrem em fêmeas com menor ganho de peso, no último mês da gestação.

\section{Agradecimentos}

Ao Conselho Nacional de Desenvolvimento Científico e Tecnológico por bolsa concedida. À Empresa Marfrig Group por ceder as instalações da Granja Carrol's para a execução do estudo e à sua equipe de trabalho, pelo auxílio prestado.

\section{Referências}

AMARAL FILHA, W.S.; BERNARDI, M.L.; WENTZ, I.; BORTOLOZZO, F.P. Reproductive performance of gilts according to growth rate and backfat thickness at mating. Animal Reproduction Science, v.121, p.139-144, 2010.

BARNETT, J.L.; CRONIN, G.M.; MCCALLUM, T.H.; NEWMAN, E.A. Effects of pen size/shape and design on aggression when grouping unfamiliar adult pigs. Applied Animal Behaviour Science, v.36, p.111-122, 1993.

HULBERT, L.E.; MCGLONE, J.J. Evaluation of drop versus trickle-feeding systems for crated or group-penned gestating sows. Journal of Animal Science, v.84, p. 1004-1014, 2006.

JANSEN, J.; KIRKWOOD, R.N.;ZANELLA,A.J.; TEMPELMAN, R.J. Influence of gestation housing on sow behavior and fertility. Journal of Swine Health and Production, v.15, p.132-136, 2007.

KRANENDONK, G.; HOPSTER, H.; FILLERUP, M.; EKKEL, E.D.; MULDER, E.J.H.; WIEGANT, V.M.; TAVERNE, M.A.M. Lower birth weight and attenuated adrenocortical response to ACTH in offspring from sows that orally received cortisol during gestation. Domestic Animal Endocrinology, v.30, p.218-238, 2006.

KRANENDONK, G.; VAN DER MHEEN, H.; FILLERUP, M.; HOPSTER, H. Social rank of pregnant sows affects their body weight gain and behavior and performance of the offspring. Journal of Animal Science, v.85, p.420-429, 2007.

LØVENDAHL, P.; DAMGAARD, L.H.; NIELSEN, B.L.; THODBERG, K.; SU, G.; RYDHMER, L. Aggressive behaviour of sows at mixing and maternal behaviour are heritable and genetically correlated traits. Livestock Production Science, v.93, p.73-85, 2005.

LUCIA, T.; DIAL, G.D.; MARSH, W.E. Lifetime reproductive performance in female pigs having distinct reasons for removal. Livestock Production Science, v.63, p.213-222, 2000. 
MARÍA LEVRINO, G.A.; VILLARROEL ROBINSON, M. Welfare status of commercial sows in three housing systems in Spain. Archieves of Zootecnie, v.52, p.453-462, 2003.

MCPHERSON, R.L.; JI, F.; WU, G.; BLANTON JUNIOR, J.R.; KIM, S.W. Growth and compositional changes of fetal tissues in pigs. Journal of Animal Science, v.82, p.2534-2540, 2004.

MILLER, H.M.; FOXCROFT, G.R.; AHERNE, F.X. Increasing food intake in late gestation improved sow condition throughout lactation but did not affect piglet viability or growth rate. Animal Science, v.71, p.141-148, 2000.

NATIONAL RESEARCH COUNCIL. Nutrient requirements of swine. $10^{\text {th }}$ rev.ed. Washington: National Academic, 1998. 211p.

NOBLET, J.; DOURMAD, J.Y.; ETIENNE, M. Energy utilization in pregnant and lactating sows: modeling of energy requirements. Journal of Animal Science, v.68, p.562-572, 1990.

NOBLET, J.; DOURMAD, J.Y.; ETIENNE, M.; LE DIVIDICH, J. Energy metabolism in pregnant sows and newborn pigs. Journal of Animal Science, v.75, p.2708-2714, 1997.

PETTIGREW JUNIOR, J.E. Supplemental dietary fat for peripartal sows: a review. Journal of Animal Science, v.53, p.107-117, 1981.

PLUSKE, J.R.; WILLIAMS, I.H.; CEGIELSKI, A.C.; AHERNE, F. X. Stomach cannulation of pregnant gilts for nutrition studies during lactation. Canadian Journal of Animal Science, v.75, p.497-500, 1995.

QUINIOU, N.; DAGORN, J.; GAUDRE, D. Variation of piglets' birth weight and consequences on subsequent performance. Livestock Production Science, v.78, p.63-70, 2002.
REMIENCE, V.; WAVREILLE, J.; CANART, B.; MEUNIER-SALAÜN, M.S.; NICKS, B.; VANDENHEEDE, M. Effects of space allowance on the welfare of dry sows kept in dynamic groups and fed with an electronic sow feeder. Applied Animal Behaviour Science, v.112, p.284-296, 2008.

SALAK-JOHNSON, J.L.; NIEKAMP, S.R.; RODRIGUEZ-ZAS, S.L.; ELLIS, M.; CURTIS, S.E. Space allowance for dry, pregnant sows in pens: body condition, skin lesions, and performance. Journal of Animal Science, v.85, p.1758-1769, 2007.

SAS INSTITUTE. Statistical analysis system. Version 9.1.3. Cary: SAS Institute, 2005.

SÉGUIN, M.J.; BARNEY, D.; WIDOWSKI, T.M. Assessment of a group-housing system for gestation sows: effects of space allowance and pen size on the incidence of superficial skin lesions, changes in body condition, and farrowing performance. Journal of Swine Health and Production, v.14, p.89-96, 2006.

SPOOLDER, H.A.M.; GEUDEKE, M.J.; VAN DER PEET-SCHWERING, C.M.C.; SOEDE, N.M. Group housing of sows in early pregnancy: a review of success and risk factors. Livestock Science, v.125, p.1-14, 2009.

WIEGAND, R.M.; GONYOU, H.W.; CURTIS, S.E. Pen shape and size: effects on pig behavior and performance. Applied Animal Behaviour Science, v.39, p.49-61, 1994.

YOUNG, M.G.; TOKACH, M.D.; AHERNE, F.X.; MAIN, R.G.; DRITZ, S.S.; GOODBAND, R.D.; NELSSEN, J.L. Effect of sow parity and weight at service on target maternal weight and energy for gain in gestation. Journal of Animal Science, v.83, p.255-261, 2005.

Recebido em 13 de janeiro de 2011 e aprovado em 20 de junho de 2011 